In review in

IEEE Transactions on Wireless Communications

\title{
A Transmit MIMO Scheme with Frequency Domain Pre- equalization for Wireless Frequency Selective Channels ${ }^{1}$
}

\author{
Ruly Lai-U Choi, and Ross D. Murch ${ }^{2}$ \\ Department of Electrical \& Electronic Engineering \\ The Hong Kong University of Science \& Technology \\ Clear Water Bay, Kowloon, \\ Hong Kong \\ Email: eeluchoi@ee.ust.hk and eermurch@ee.ust.hk
}

\begin{abstract}
In this paper, we introduce a transmit MIMO scheme with frequency domain preequalization for a multipath or frequency selective channel. In this scheme, MIMO processing in the frequency domain is performed at the transmitter or base station so that the receiver or mobile station only requires limited processing. This scheme provides high data rates and also inherits from the frequency domain equalization the property of relatively low complexity in severe multipath environments. The MIMO transmit processing is derived by minimizing the mean square errors (MMSE), and expressions for the signal to interference-plus-noise ratio (SINR) and error probability based on the Gaussian approximation of the interference term are provided. Some important associated issues, such as channel errors and computational complexity, are also investigated. Numerical simulations are also provided and these demonstrate the improved performance of our proposed scheme compared to other transmit MIMO schemes. In particular, they show that the proposed system can attain multipath or frequency diversity of the channel.
\end{abstract}

\footnotetext{
${ }^{1}$ This work was supported by the Hong Kong Research Grant Council (HKUST6024/01E).

${ }^{2}$ Corresponding Author. Email: eermurch@ee.ust.hk. Phone: (+852) 23587044. Fax: (+852) 23581485 .
} 


\section{Introduction}

Recently, high data rate communication has gained a lot of attention because of the rapid growth of wireless voice subscribers, the Internet, and the use of portable computing devices. A fundamental challenge in transmitting high data rates over radio channels is to overcome the signal corruption caused by multipath propagation within a limited available bandwidth.

Multipath dispersion causes intersymbol interference (ISI) in data transmission, where the number of symbols of dispersion grows linearly with the data rate. Frequency-domain equalization (FDE) has been proposed as a technique to combat ISI [1], [2] and the advantages of this technique include low peak-to-average power ratio (PAPR) and the property of relatively low complexity growth with the number of symbols of dispersion. It has also been adopted as an option in the Fixed Wireless broadband IEEE 802.16 standard for operating in the frequency band from 2 to 11 $\mathrm{GHz}[3]$.

On the other hand, multiple-input multiple-output (MIMO) systems, with multiple antennas employed at both the transmitter and receiver, promise improved performance and bandwidth efficiency compared to conventional systems [4], and therefore provide a solution when limited bandwidth is available. In general, MIMO wireless systems can be classified into three approaches. The first approach is where processing associated with the MIMO signals is performed only at the receiver as in V-BLAST, MLD, and MMSE [5]-[7]. In these systems multiple parallel data streams are transmitted directly into the wireless channel, with the task of retrieving the individual signals resting on MIMO signal processing at the receiver. The second approach is where MIMO signal pre-processing is utilized before transmission, in addition to MIMO signal processing at the receiver. This approach includes SVD based MIMO techniques where antenna weights and processing is utilized at both the transmitter and receiver [8] and other joint transmit and receive processing (e.g. [9]). A third approach is also possible where MIMO signal pre-processing, before transmission, is utilized only so that a simplified receiver structure or mobile station can be devised. The receiver or mobile station still requires multiple front-ends but the associated MIMO signal processing at the receiver is greatly reduced because 
appropriate MIMO processing has already been performed before transmission. We call this approach transmit MIMO. An important potential application for transmit MIMO techniques is in the downlink of wireless communication systems with $\mathrm{V}$ BLAST or similar techniques utilized in the uplink, creating a high-speed duplex system with a simplified mobile station transceiver structure.

In this paper, we introduce a new transmit MIMO scheme with MIMO frequency domain pre-equalization and simplified receivers to provide high data rates to combat ISI and fading for MIMO wireless systems. Previous work on transmit MIMO systems with signal pre-processing before transmission and with a simplified receiver structure includes transmit maximum ratio combining (MRC) combining [10], [11], and transmit zero forcing scheme [12], [13]. However, the transmit MRC scheme has to be utilized with other multiple access techniques, such as code division multiple access (CDMA), for MIMO systems because the scheme itself cannot deal with the interference. On the other hand, the transmit zero forcing scheme is proposed for the flat fading channel and therefore, it has to be associated with orthogonal frequency division modulation (OFDM) in a multipath or frequency selective channel. The idea of transmission in frequency domain has previously been studied in [14]. It has been shown that this approach is asymptotically optimum from the information theory point of view. However, note that the system discussed in [14] is actually signal processing in an OFDM system. Also, in order to achieve the capacity, water filling solution and both transmit and receive processing has to be applied. Conventionally, block processing (in time succession) is utilized to deal with the frequency selective channels (e.g. [8] and [9]). The computational complexity is very high because the size of the transmit matrix is dependent on the data block length (e.g. in the order of 3 of the block length in [8] and [9]). Simplification of the complexity is necessary.

Our work is different from previous results in that we introduce a transmit MIMO scheme with frequency domain pre-equalization for a multipath or frequency selective channel. In this scheme, MIMO processing in the frequency domain is performed at the transmitter or base station. It inherits from FDE the property of relatively low complexity in severe multipath environments and this provides a solution to simplify the complexity of the conventional block processing (in time succession). Another characteristic of this scheme is that it utilizes a simplified receiver structure and this makes it applicable to a multi-user MISO (multiple-input single-output) system as 
well. The optimal transmit frequency domain coefficient matrix is derived by minimizing the mean square errors (MMSE). Then, in order to have a better understanding of the system, we also derive an expression for the signal to interference-plus-noise ratio (SINR) and error probability based on the Gaussian approximation of the interference term. The Gaussian approximation is not always good, but evidence shows that relatively accurate bit error rate estimates can be obtained in this configuration. Furthermore, some important associated issues, such as channel errors and computational complexity, are investigated. Numerical simulations are also provided and demonstrate the performance of our proposed scheme. In particular, it shows that the proposed system can obtain multipath or frequency diversity of the channel.

One disadvantage of transmit processing technique is the requirement for accurate channel knowledge and restricts us to systems where channel state information is available. These systems include time division duplex (TDD) systems (such as IEEE 802.16, IEEE 802.11a, and HiperLAN/II) where the up and downlink channels are reciprocal and systems where there is an option for passing channel information to the transmitter. Our transmit processing technique may not be useful in a frequency division duplex (FDD) system because of the requirement for accurate channel feedback. The peak-to-average power ratio at the transmitter of our proposed system is the same as that at the transmitter of an OFDM system. Therefore, high linear power amplifier is also required at the transmitter, which is another disadvantage.

This paper is organized as follows. In Section II, the system model is first introduced. Then, in Section III the optimal transmit frequency domain coefficient matrix is derived. Section IV gives an expression for the SINR and provides a Gaussian approximation for the bit error probability. After that, some associated issues are investigated in Section V and some numerical results and comparisons are provided in Section VI. Finally, Section VII concludes our work.

\section{System Model}

The configuration of our proposed transmit MIMO scheme is shown in Figure 1, where $M$ antennas are located at the transmitter and $K$ antennas are located at the receiver. In this paper, we assume $K \leq M$. At the transmitter, the data stream is demultiplexed into $K$ sub-streams which go through some transmit processing as 
shown in Figure 2. Each of the sub-streams first passes through a serial-to-parallel converter (S/P), a Fast Fourier Transform (FFT) and then are transformed by the transmit frequency domain pre-equalization (preFDE) coefficient matrix, $\mathbf{T}^{(k)}$. After that, at each transmit antenna the signals of the $K$ sub-streams add together and pass through an Inverse Fast Fourier Transform (IFFT) followed by a parallel-to-serial converter $(\mathrm{P} / \mathrm{S})$. A cyclic prefix is appended to the signals before they are launched to the channel.

At the receiver (see Figure 1), each receive antenna or front-end is dedicated to receive one data spatial sub-stream without the need for further MIMO signal processing. The cyclic prefix is removed and a gain a common to all received data streams is included, which can be thought of as being part of the automatic gain control utilized to normalize the received signals to some fixed threshold for demodulation.

A complex baseband discrete model is assumed throughout this paper. The antenna elements transmit or receive information through a wireless communication channel, which is here characterized by a multipath or frequency selective fading channel. We utilize the simplified tapped delay line multipath channel model of [15], and assume an arbitrary power delay profile with maximum excess delay $T_{\max }$. Also, we define the number of paths $L=\left\lfloor T_{\max } / T\right\rfloor+1$, where $T$ is the symbol duration and $\lfloor x\rfloor$ represents the largest integer less than or equal to $x$. Hence, for a particular channel, say the channel between the $m$-th transmit antenna and the $k$-th receive antenna, the complex low-pass impulse response of the channel can be written as

$$
h^{(k, m)}(t)=\sum_{l=0}^{L-1} h_{l}^{(k, m)} \delta(t-l T),
$$

where the superscript $m$ and $k$ refer to the $m$-th transmit antenna and the $k$-th receive antenna, respectively. The subscript $l$ refers to the $l$-th path of the channel. The coefficient $h_{l}^{(k, m)}$ is a zero-mean, circularly symmetric, complex Gaussian distributed random gain at the time instant $l T$, and we assume that $\left\{h_{l}^{(k, m)}\right\}$ are uncorrelated for all $m, k$, and $l$. Throughout this paper, the average squared gain of each link from one transmit antenna to one receive antenna is normalized. That is, $\mathrm{E}\left[\sum_{l=0}^{L-1}\left\|h_{l}^{(k, m)}\right\|^{2}\right]=1$, where $\|\cdot\|$ represents the Euclidean norm operation and E[.] represents the expectation operation. $\delta(t)$ is the delta function.

Single-carrier block transmission of block length $N$ for each sub-stream over a 
slow fading frequency selective channel is considered and the data block of the $k$-th sub-stream is denoted as $\mathbf{x}^{(k)}=\left[\begin{array}{llll}x_{1}^{(k)} & x_{2}^{(k)} & \cdots & x_{N}^{(k)}\end{array}\right]^{T}$, where the superscript ${ }^{T}$ represents the transpose operation. Note that the superscripts in the bracket denote the spatial index, while the subscripts denotes the time index. We assume that channel information is known at the transmitter and the channel does not change for the duration of a block of data. Each data block is appended with a length- $P$ cyclic prefix to eliminate interblock interference. This is achieved by discarding the first $P$ received symbols corresponding to the cyclic prefix [15]. Hence, out of every $(N+P)$ received symbols, only $N$ symbols are processed. We let the $N \times N$ matrix $\mathbf{W}$ represent the FFT matrix whose $(n, k)$ element is given by $W_{n, k}=1 / \sqrt{N} e^{j 2 \pi(n-1)(k-1) / N}$, in which $1 \leq n, k \leq N$, and $\mathbf{W}^{H}$ represent the IFFT matrix. $\mathbf{T}^{(i)}$ is the $M N \times N$ transmit frequency domain coefficient matrix for the $i$-th sub-stream. $\mathbf{H}^{(k, m)}$ is the $N \times N$ circulant channel matrix between the $m$-th transmit antenna and the $k$-th receive antenna, whose first column is equal to the channel impulse response (CIR) appended by $N-L$ zeros. That is,

$$
\mathbf{H}^{(k, m)}=\left[\begin{array}{ccccccc}
h_{0}^{(k, m)} & 0 & \cdots & & & \cdots & h_{1}^{(k, m)} \\
h_{1}^{(k, m)} & h_{0}^{(k, m)} & \ddots & \cdots & \cdots & & \vdots \\
\vdots & h_{1}^{(k, m)} & \ddots & & & \ddots & h_{L-1}^{(k, m)} \\
h_{L-1}^{(k, m)} & \vdots & \ddots & & & & 0 \\
\mathbf{0} & \ddots & & & & \ddots & \vdots \\
\vdots & \ddots & \ddots & \cdots & \cdots & \ddots & \mathbf{0} \\
0 & \cdots & & & & \cdots & h_{0}^{(k, m)}
\end{array}\right] .
$$

Also we let $\mathbf{R}^{(k)}=a \mathbf{I}$ represent the receiver at the $k$-th receive antenna, in which $\mathbf{I}$ is an $N \times N$ identity matrix and $a$ is a scalar. The output block of signals for the $k$-th sub-stream is denoted as $\mathbf{y}^{(k)}=\left[\begin{array}{llll}y_{1}^{(k)} & y_{2}^{(k)} & \cdots & y_{N}^{(k)}\end{array}\right]^{T}$. Accordingly, the input-output relationship for the $k$-th sub-stream can be expressed in matrix form as

$$
\mathbf{y}^{(k)}=\mathbf{R}^{(k)} \sum_{i=1}^{K}\left[\begin{array}{llll}
\mathbf{H}^{(k, 1)} \mathbf{W}^{H} & \mathbf{H}^{(k, 2)} \mathbf{W}^{H} & \cdots & \mathbf{H}^{(k, M)} \mathbf{W}^{H}
\end{array}\right] \mathbf{T}^{(i)} \mathbf{W} \mathbf{x}^{(i)}+\mathbf{R}^{(k)} \mathbf{n}^{(k)},
$$

where $\mathbf{n}^{(k)}=\left[\begin{array}{llll}n_{1}^{(k)} & n_{2}^{(k)} & \cdots & n_{N}^{(k)}\end{array}\right]^{T}$ is a length- $N$ block of noise, whose elements are i.i.d. zero-mean, circularly symmetric, complex Gaussian distributed with real and imaginary part variance of $0.5 \sigma^{2}$. The superscript $H$ represents the complex conjugate transpose operation. The input data and noise are assumed to be uncorrelated. Also, it is assumed that the input data are zero-mean and circularly symmetric complex random variables with unity variance (real and imaginary part 
variance of 0.5). Furthermore, we can write the decision statistics of the $K$ substreams in a vector form as $\mathbf{y}=\left[\begin{array}{llll}\mathbf{y}^{(1) T} & \mathbf{y}^{(2) T} & \cdots & \mathbf{y}^{(K) T}\end{array}\right]^{T}$, which can be written as

$$
\mathbf{y}=\mathbf{R} \widetilde{\mathbf{H}} \mathbf{T} \tilde{\mathbf{W}} \mathbf{x}+\mathbf{R n}
$$

where

$$
\begin{gathered}
\mathbf{x}=\left[\begin{array}{llll}
\mathbf{x}^{(1) T} & \mathbf{x}^{(2) T} & \cdots & \mathbf{x}^{(K) T}
\end{array}\right]^{T}, \\
\mathbf{n}=\left[\begin{array}{llll}
\mathbf{n}^{(1) T} & \mathbf{n}^{(2) T} & \cdots & \mathbf{n}^{(K) T}
\end{array}\right]^{T}, \\
\mathbf{T}=\left[\begin{array}{llll}
\mathbf{T}^{(1)} & \mathbf{T}^{(2)} & \cdots & \mathbf{T}^{(K)}
\end{array}\right], \\
\mathbf{R}=\left[\begin{array}{cccc}
\mathbf{R}^{(1)} & \mathbf{0} & \cdots & \mathbf{0} \\
\mathbf{0} & \mathbf{R}^{(2)} & \ddots & \vdots \\
\vdots & \ddots & \ddots & \mathbf{0} \\
\mathbf{0} & \cdots & \mathbf{0} & \mathbf{R}^{(K)}
\end{array}\right], \\
\widetilde{\mathbf{H}}=\left[\begin{array}{cccc}
\mathbf{H}^{(1,1)} \mathbf{W}^{H} & \mathbf{H}^{(1,2)} \mathbf{W}^{H} & \cdots & \mathbf{H}^{(1, M)} \mathbf{W}^{H} \\
\mathbf{H}^{(2,1)} \mathbf{W}^{H} & \mathbf{H}^{(2,2)} \mathbf{W}^{H} & \cdots & \mathbf{H}^{(2, M)} \mathbf{W}^{H} \\
\vdots & \vdots & & \ddots \\
\mathbf{H}^{(K, 1)} \mathbf{W}^{H} & \mathbf{H}^{(K, 2)} \mathbf{W}^{H} & \cdots & \mathbf{H}^{(K, M)} \mathbf{W}^{H}
\end{array}\right],
\end{gathered}
$$

and

$$
\widetilde{\mathbf{W}}=\left[\begin{array}{cccc}
\mathbf{W} & \mathbf{0} & \cdots & \mathbf{0} \\
\mathbf{0} & \mathbf{W} & \ddots & \vdots \\
\vdots & \ddots & \ddots & \mathbf{0} \\
\mathbf{0} & \cdots & \mathbf{0} & \mathbf{W}
\end{array}\right]
$$

\section{Transmit Pre-equalization Weight}

Our objective is to find the overall coefficient matrix $\mathbf{T}$ in (4) to minimize the mean square errors between the output and input under the constraint that the transmit power is fixed. Mathematically, our problem expression can be written as

$$
\mathbf{T}=\arg \min _{\operatorname{trace}\left(\mathbf{T}^{H} \mathbf{T}\right)=K N P_{s}} \mathrm{E}\left[\|\mathbf{y}-\mathbf{x}\|^{2}\right]
$$

where trace(.) represents the trace operation. The constraint, $\operatorname{trace}\left(\mathbf{T}^{H} \mathbf{T}\right)=K N P_{s}$, provides the total transmit power constraint and $P_{s}$ is the average transmit power per symbol. From this constraint, we can obtain $\operatorname{trace}\left(\mathbf{T}^{H} \mathbf{T}\right) /(K N P)=1$, which is also equivalent to $\mathrm{E}\left[\|\mathbf{T} \tilde{\mathbf{W}} \mathbf{x}\|^{2}\right] /\left(K N P_{s}\right)=1$. By substituting (4) into (11) and letting $\mathbf{S}=a \mathbf{T}$, it can be shown that 


$$
\mathbf{S}=\arg \min _{a^{2}=\frac{t \operatorname{tace}\left(\mathbf{S}^{H} \mathbf{S}\right)}{K N P_{s}}}\left\{\mathrm{E}\left[\|\widetilde{\mathbf{H}} \mathbf{S} \widetilde{\mathbf{W}} \mathbf{x}-\mathbf{x}\|^{2}\right]+\frac{\sigma^{2}}{P_{s}} \mathrm{E}\left[\|\mathbf{S} \widetilde{\mathbf{W}} \mathbf{x}\|^{2}\right]\right\} .
$$

Since the symbols in $\mathbf{x}$ are i.i.d. zero mean complex random variables with unity variance, the cost function in the parentheses $\{$.$\} in .(12) can be simplified to$

$$
\mathrm{C}(\mathbf{S} \widetilde{\mathbf{W}})=\operatorname{trace}\left[(\tilde{\mathbf{H}} \mathbf{S} \tilde{\mathbf{W}}-\mathbf{I})(\widetilde{\mathbf{H}} \mathbf{S} \tilde{\mathbf{W}}-\mathbf{I})^{H}\right]+\frac{\sigma^{2}}{P_{s}} \operatorname{trace}\left[(\mathbf{S} \widetilde{\mathbf{W}})(\mathbf{S} \tilde{\mathbf{W}})^{H}\right] .
$$

By letting $\widetilde{\mathbf{S}}=\mathbf{S} \widetilde{\mathbf{W}}$ and taking $\nabla_{\widetilde{\mathbf{s}}} \mathrm{C}(\widetilde{\mathbf{S}})=0$, we can obtain the solution to the problem formulated in (12) as

$$
\mathbf{S} \widetilde{\mathbf{W}}=\left(\widetilde{\mathbf{H}}{ }^{H} \widetilde{\mathbf{H}}+\frac{\sigma^{2}}{P_{s}} \mathbf{I}\right)^{-1} \widetilde{\mathbf{H}}^{H}=\widetilde{\mathbf{H}}^{H}\left(\widetilde{\mathbf{H}} \widetilde{\mathbf{H}}^{H}+\frac{\sigma^{2}}{P_{s}} \mathbf{I}\right)^{-1} .
$$

Multiplying (14) by $\widetilde{\mathbf{W}}^{H}$, this becomes

$$
\mathbf{S}=\left[(\tilde{\mathbf{W}} \widetilde{\mathbf{H}})^{H}(\tilde{\mathbf{W}} \widetilde{\mathbf{H}})+\frac{\sigma^{2}}{P_{s}} \mathbf{I}\right]^{-1}(\widetilde{\mathbf{W}} \widetilde{\mathbf{H}})^{H}=(\tilde{\mathbf{W}} \widetilde{\mathbf{H}})^{H}\left[(\widetilde{\mathbf{W}} \widetilde{\mathbf{H}})(\tilde{\mathbf{W}} \widetilde{\mathbf{H}})^{H}+\frac{\sigma^{2}}{P_{s}} \mathbf{I}\right]^{-1}
$$

Simplification occurs by letting $\mathbf{D}^{(k, m)}=\mathbf{W} \mathbf{H}^{(k, m)} \mathbf{W}^{H}$ and noting that $\mathbf{D}^{(k, m)}$ is a diagonal matrix with diagonal elements $D_{n, n}^{(k, m)}=\sum_{l=0}^{L-1} h_{l}^{(k, m)} W_{n, l}$ for $n=1, \ldots, N$ (since $\mathbf{H}^{(k, m)}$ is a circulant channel matrix), which is the FFT frequency response of the frequency selective channel. Hence,

$$
\widetilde{\mathbf{W}} \widetilde{\mathbf{H}}=\left[\begin{array}{cccc}
\mathbf{D}^{(1,1)} & \mathbf{D}^{(1,2)} & \cdots & \mathbf{D}^{(1, M)} \\
\mathbf{D}^{(2,1)} & \mathbf{D}^{(2,2)} & \cdots & \mathbf{D}^{(2, M)} \\
\vdots & \vdots & \ddots & \vdots \\
\mathbf{D}^{(K, 1)} & \mathbf{D}^{(K, 2)} & \cdots & \mathbf{D}^{(K, M)}
\end{array}\right],
$$

and therefore

$$
\mathbf{S}=\left[\begin{array}{cccc}
\mathbf{S}^{(1,1)} & \mathbf{S}^{(2,1)} & \cdots & \mathbf{S}^{(K, 1)} \\
\mathbf{S}^{(1,2)} & \mathbf{S}^{(2,2)} & \cdots & \mathbf{S}^{(K, 2)} \\
\vdots & \vdots & \ddots & \vdots \\
\mathbf{S}^{(1, M)} & \mathbf{S}^{(2, M)} & \cdots & \mathbf{S}^{(K, M)}
\end{array}\right]
$$

where $\mathbf{S}^{(k, m)}$ is an $N \times N$ matrix, whose elements are (substitute (16) into (15))

$$
S_{n, j}^{(k, m)}=0 \text { for } n \neq j
$$

and $S_{n, n}^{(k, m)}$ given by

$$
\mathbf{S}_{n}=\left(\mathbf{D}_{n}{ }^{H} \mathbf{D}_{n}+\frac{\sigma^{2}}{P_{s}} \mathbf{I}\right)^{-1} \mathbf{D}_{n}{ }^{H}=\mathbf{D}_{n}{ }^{H}\left(\mathbf{D}_{n} \mathbf{D}_{n}{ }^{H}+\frac{\sigma^{2}}{P_{S}} \mathbf{I}\right)^{-1}
$$

where, for every tone $n(1 \leq n \leq N)$, we have 


$$
\mathbf{S}_{n}=\left[\begin{array}{cccc}
S_{n, n}^{(1,1)} & S_{n, n}^{(2,1)} & \cdots & S_{n, n}^{(K, 1)} \\
S_{n, n}^{(1,2)} & S_{n, n}^{(2,2)} & \cdots & S_{n, n}^{(K, 2)} \\
\vdots & \vdots & \ddots & \vdots \\
S_{n, n}^{(1, M)} & S_{n, n}^{(2, M)} & \cdots & S_{n, n}^{(K, M)}
\end{array}\right]
$$

and

$$
\mathbf{D}_{n}=\left[\begin{array}{cccc}
D_{n, n}^{(1,1)} & D_{n, n}^{(1,2)} & \cdots & D_{n, n}^{(1, M)} \\
D_{n, n}^{(2,1)} & D_{n, n}^{(2,2)} & \cdots & D_{n, n}^{(2, M)} \\
\vdots & \vdots & \ddots & \vdots \\
D_{n, n}^{(K, 1)} & D_{n, n}^{(K, 2)} & \cdots & D_{n, n}^{(K, M)}
\end{array}\right] .
$$

From the power constraint, $\operatorname{trace}\left(\mathbf{T}^{H} \mathbf{T}\right)=K N P_{s}$, we can obtain

$$
a=\sqrt{\frac{\operatorname{trace}\left(\mathbf{S}^{H} \mathbf{S}\right)}{K N P_{s}}}=\sqrt{\frac{\sum_{k=1}^{K} \sum_{m=1}^{M} \sum_{n=1}^{N}\left\|S_{n, n}^{(k, m)}\right\|^{2}}{K N P_{s}}}
$$

and since $\mathbf{S}=a \mathbf{T}$, we then obtain

$$
\mathbf{T}=\frac{1}{a} \mathbf{S}
$$

Notice that there is no dependency between signals of different carrier frequencies in the frequency domain pre-equalization because $S_{n, j}^{(k, m)}=0$ for $n \neq j$. Therefore, the transmit coefficient matrix for a particular sub-stream, $\mathbf{T}^{(k)}$, can be decomposed into $N$ independent weight vectors of length $M$ for the $N$ sub-carriers. As shown in Figure 3, each sub-carrier signal is multiplied by a weight vector of length $M$ and generates $M$ signals, each of which is for a transmit antenna. We refer to this transmit technique as Transmit Optimized MIMO with Frequency Domain Pre-equalization (MIMO-preFDE).

A close observation of (19) reveals that when the noise variance is large or the effect of noise is dominant, the matrix $\mathbf{S}_{n}$ becomes proportional to the complex conjugate of the channel frequency response $\mathbf{D}_{n}$, which is similar to a transmit maximum ratio combining (MRC) scheme [10], [11]. When the noise variance is small or the effect of noise is negligible, the matrix $\mathbf{S}_{n}$ becomes proportional to the pseudoinverse of the channel frequency response $\mathbf{D}_{n}$, which is similar to the transmit Zero Forcing scheme [13], [21]. Therefore, we can expect our proposed scheme to outperform both the transmit MRC and the transmit Zero Forcing schemes. Finally, we would like to emphasize that our proposed transmit scheme performs a spacefrequency processing, which optimises across all frequency subcarriers and spatial 
antennas.

Finally, to compare our scheme with the well-known SVD with water filling optimization [14], in which transmit processing and receive processing have to used jointly, our transmit scheme mainly concentrates the complexity at the transmitter. Although our scheme may not be able to reach the capacity of the water filling optimization, the water filling optimization has to be used with adaptive modulation. Otherwise, the BER performance may be worse than our scheme because the BER is dominated by the channel with the smallest eigenvalue. To compare our scheme with the joint transmit and receive scheme in [8] and [9], in which transmit and receive block processing (in time succession) is utilized to deal with frequency selective channels, the computational complexity of our scheme is much lower because of the special proposed transmit structure and the simplified receiver structure. Also, note that the simplified receiver structure makes our proposed scheme applicable to a multi-user MISO system as well.

\section{Performance Analysis}

In this section, we derive an expression for the signal to interference-plus-noise ratio (SINR) and error probability based on the Gaussian approximation of the interference term. Such an approximation is not always good, but we will show that relatively accurate bit error rate estimates can be obtained in this case. We use this result later to provide a better understanding of our technique.

By substituting (23) into (3), it can be shown that

$$
\mathbf{y}^{(k)}=\sum_{i=1}^{K} \mathbf{W}^{H} \mathbf{G}^{(k, i)} \mathbf{W} \mathbf{x}^{(i)}+a \mathbf{n}^{(k)}
$$

where $\mathbf{G}^{(k, i)}$ is an $N \times N$ diagonal matrix and $\mathbf{G}^{(k, i)}=\sum_{m=1}^{M} \mathbf{D}^{(k, m)} \mathbf{S}^{(i, m)}$. Therefore, the $n$-th diagonal element is $G_{n, n}^{(k, i)}=\sum_{m=1}^{M} D_{n, n}^{(k, m)} S_{n, n}^{(i, m)}$. For a particular symbol, say the $n_{0}$-th symbol of the data block of the $k_{0}$-th sub-stream, we can express the input and output relationship as

$$
y_{n_{0}}^{\left(k_{0}\right)}=\sum_{k=1}^{K} \sum_{i=1}^{N} G_{i, i}^{\left(k_{0}, k\right)} W_{i, n_{0}}^{H} \sum_{j=1}^{N} W_{i, j} x_{j}^{(k)}+a n_{n_{0}}^{\left(k_{0}\right)}=d_{n_{0}}^{\left(k_{0}\right)}+I_{n_{0}}^{\left(k_{0}\right)}+v_{n_{0}}^{\left(k_{0}\right)}
$$

where $d_{n_{0}}^{\left(k_{0}\right)}, I_{n_{0}}^{\left(k_{0}\right)}$ and $v_{n_{0}}^{\left(k_{0}\right)}$ represent the desired signal term, the interference term and the noise term, respectively, and can be given by 


$$
\begin{gathered}
d_{n_{0}}^{\left(k_{0}\right)}=\frac{1}{N} \sum_{i=1}^{N} G_{i, i}^{\left(k_{0}, k_{0}\right)} x_{n_{0}}^{\left(k_{0}\right)}, \\
I_{n_{0}}^{\left(k_{0}\right)}=\sum_{i=1}^{N} G_{i, i}^{\left(k_{0}, k_{0}\right)} W_{i, n_{0}}^{H} \sum_{j=1, j \neq n_{0}}^{N} W_{i, j} x_{j}^{\left(k_{0}\right)}+\sum_{k=1, k \neq k_{0}}^{K} \sum_{i=1}^{N} G_{i, i}^{\left(k_{0}, k\right)} W_{i, n_{0}}{ }^{H} \sum_{j=1}^{N} W_{i, j} x_{j}^{(k)},
\end{gathered}
$$

and

$$
v_{n_{0}}^{\left(k_{0}\right)}=a n_{n_{0}}^{\left(k_{0}\right)} .
$$

Note that in (27) the first term on the right hand side is the self interference and the second term is the interference from other sub-streams. Since the data symbols in $\mathbf{x}$ and the elements in the noise vector $\mathbf{n}$ are complex, zero-mean, and uncorrelated with variances 1 and $\sigma^{2}$, respectively, it can be shown that the first moment of the desired signal term, the interference term and the noise term conditioned on the channel is equal to zero, respectively, and the second moment is given by

$$
\begin{gathered}
\mathrm{E}\left[\left\|d_{n_{0}}^{\left(k_{0}\right)}\right\|^{2} \mid \mathbf{H}\right]=\frac{1}{N^{2}}\left\|\sum_{i=1}^{N} G_{i, i}^{\left(k_{0}, k_{0}\right)}\right\|^{2}, \\
\mathrm{E}\left[\left\|I_{n_{0}}^{\left(k_{0}\right)}\right\|^{2} \mid \mathbf{H}\right]=\frac{1}{N} \sum_{k=1}^{K} \sum_{i=1}^{N}\left\|G_{i, i}^{\left(k_{0}, k\right)}\right\|^{2}-\frac{1}{N^{2}}\left\|\sum_{i=1}^{N} G_{i, i}^{\left(k_{0}, k_{0}\right)}\right\|^{2},
\end{gathered}
$$

and

$$
\mathrm{E}\left[\left\|v_{n_{0}}^{\left(k_{0}\right)}\right\|^{2} \mid \mathbf{H}\right]=a^{2} \sigma^{2} .
$$

Accordingly, the receive signal to interference-plus-noise ratio (SINR) conditioned on the channel, $\mathbf{H}$, can be given by

$$
\gamma_{n_{0}}^{\left(k_{0}\right)}=\frac{\frac{1}{N^{2}}\left\|\sum_{i=1}^{N} G_{i, i}^{\left(k_{0}, k_{0}\right)}\right\|^{2}}{\frac{1}{N} \sum_{k=1}^{K} \sum_{i=1}^{N}\left\|G_{i, i}^{\left(k_{0}, k\right)}\right\|^{2}-\frac{1}{N^{2}}\left\|\sum_{i=1}^{N} G_{i, i}^{\left(k_{0}, k_{0}\right)}\right\|^{2}+a^{2} \sigma^{2}} .
$$

Note that the SINR conditioned on the channel does not depend on $n_{0}$. Therefore, the output SINR is the same for the symbols in a particular data block and the subscript $n_{0}$ can be dropped. For convenience, we denote $\gamma^{\left(k_{0}\right)}=\gamma_{n_{0}}^{\left(k_{0}\right)}$ in the following discussions.

If $N$ is large, we can approximate the interference as a Gaussian random variable by the Central Limit Theorem [16]. In this case, it follows that the bit error probability for QPSK given the channel is given by [15]

$$
\operatorname{Pr}(e \mid \mathbf{H})=Q\left(\sqrt{\gamma^{\left(k_{0}\right)}}\right)
$$


where $Q($.$) represents the standard Q-function and \gamma^{\left(k_{0}\right)}$ is given in (32).

When $P_{s}>>\sigma^{2}$, it can be shown that we can approximate $\mathbf{G}^{(k, k)} \approx \mathbf{I}$ and $\mathbf{G}^{(k, i)} \approx \mathbf{0}$ for $k \neq i$. Hence, the SINR can be approximated as

$$
\gamma^{\left(k_{0}\right)} \approx \frac{1}{a^{2} \sigma^{2}}=\frac{K N P_{s}}{\sigma^{2} \operatorname{trace}\left(\mathbf{S}^{H} \mathbf{S}\right)} .
$$

Furthermore, when $P_{s} \gg \sigma^{2}$, we can approximate $\operatorname{trace}\left(\mathbf{S}^{H} \mathbf{S}\right) \approx \operatorname{trace}\left(\left(\mathbf{D D}^{H}\right)^{-1}\right)$, therefore the SINR can be approximated further as

$$
\gamma^{\left(k_{0}\right)} \approx \frac{K N P_{s}}{\left.\sigma^{2} \operatorname{trace}\left((\mathbf{D D})^{H}\right)^{-1}\right)}=\frac{K N P_{s}}{\sigma^{2} \operatorname{trace}\left(\left(\widetilde{\mathbf{H}} \widetilde{\mathbf{H}}^{H}\right)^{-1}\right)}=\frac{K N P_{s}}{\sigma^{2} \sum_{i} \frac{1}{\lambda_{i}}},
$$

where $\lambda_{i}$ is the eigenvalue of $\widetilde{\mathbf{H}} \widetilde{\mathbf{H}}^{H}$. An approximate upper and lower bound of the SINR is

$$
\frac{P_{s}}{\sigma^{2}} \lambda_{\min } \leq \gamma^{\left(k_{0}\right)} \leq \frac{P_{s}}{\sigma^{2}} \lambda_{\max }
$$

where $\lambda_{\max }$ and $\lambda_{\min }$ are the maximum and minimum eigenvalue of $\widetilde{\mathbf{H}} \widetilde{\mathbf{H}}^{H}$, respectively. It can be observed from (35) that the SINR is the same for all the $N$ symbols in each of $K$ sub-streams.

In order to have more insight, let us consider the special case when the number of receive antennas $K=1$ (we refer this as a multiple-input single-output (MISO) system). By using (35), it can be shown that the SINR can be approximated as

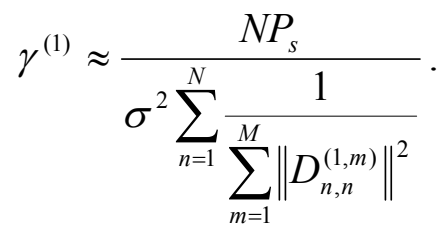

From (37), it can be seen that as the number of transmit antennas $(M)$ increases the value of SINR increases. By using the Geometry inequality, it can be shown that

$$
\sum_{n=1}^{N} \frac{1}{\sum_{m=1}^{M}\left\|D_{n, n}^{(1, m)}\right\|^{2}} \geq N\left(\prod_{n=1}^{N} \frac{1}{\sum_{m=1}^{M}\left\|D_{n, n}^{(1, m)}\right\|^{2}}\right)^{\frac{1}{N}}
$$

and therefore, 


$$
\gamma^{(1)} \leq \frac{P_{s}}{\sigma^{2}}\left(\prod_{n=1}^{N} \sum_{m=1}^{M}\left\|D_{n, n}^{(1, m)}\right\|^{2}\right)^{\frac{1}{N}} \leq \frac{P_{s}}{N \sigma^{2}} \sum_{n=1}^{N} \sum_{m=1}^{M}\left\|D_{n, n}^{(1, m)}\right\|^{2}=\frac{P_{s}}{\sigma^{2}} \sum_{m=1}^{M} \sum_{l=0}^{L-1}\left\|h_{l}^{(1, m)}\right\|^{2} .
$$

It is interesting to see that the upper bound of the SINR in (39) is equal to the signalto-noise ratio (SNR) of a maximum ratio combining (MRC) receiver, which optimally combines all the multipaths. This may indicate that we are likely to gain multipath or frequency diversity since the SINR is less than the upper bound. For a MIMO configuration, we therefore expect that diversity from the multipath or frequency and space can be obtained because the channel gain is averaged among all the sub-carriers and sub-streams. This expectation will be demonstrated by the numerical results in Section VI.

\section{Associated Issues}

In this section, we wish to address some of the associated problems and issues with our transmit MIMO scheme. This includes channel estimation and computational complexity.

\section{A. Channel Estimation}

Our technique requires accurate channel state information (CSI) of the downlink channel and this may not be obtained in practice. Therefore, the robustness of our MIMO scheme in the presence of channel estimation errors is a key issue to be addressed. Throughout this sub-section, the discussion is assumed to be in the frequency domain.

To quantify the problem, we let $D_{n, n}^{(k, m)}=\rho \widetilde{D}_{n, n}^{(k, m)}+e_{n, n}^{(k, m)}$, where $\widetilde{D}_{n, n}^{(k, m)}$ is an estimate of the true channel branch gain, $D_{n, n}^{(k, m)}$, and $e_{n, n}^{(k, m)}$ is the estimation error that is assumed uncorrelated with $\widetilde{D}_{n, n}^{(k, m)}$. The correlation coefficient, $\rho$, between $D_{n, n}^{(k, m)}$ and $\widetilde{D}_{n, n}^{(k, m)}$, is given by

$$
\rho=\frac{E\left[D_{n, n}^{(k, m)} \widetilde{D}_{n, n}^{(k, m)}\right]}{\sqrt{E\left[\left\|D_{n, n}^{(k, m)}\right\|^{2}\right] \times E\left[\left\|\widetilde{D}_{n, n}^{(k, m)}\right\|^{2}\right]}} .
$$

Notice that $\rho$ is a real number and $-1 \leq \rho \leq+1$. If we let $\sigma_{D}{ }^{2}=E\left[\left\|D_{n, n}^{(k, m)}\right\|^{2}\right]$, $\sigma_{\widetilde{D}}^{2}=E\left[\left\|\widetilde{D}_{n, n}^{(k, m)}\right\|^{2}\right]$, and ${\sigma_{D}}^{2}={\sigma_{\widetilde{D}}}^{2}$, then $\sigma_{e}{ }^{2}=\left(1-\rho^{2}\right) \sigma_{D}{ }^{2}$. We define the normalized mean square error (MSE) of the channel estimation as 


$$
\varepsilon=\frac{E\left[\left\|D_{n, n}^{(k, m)}-\widetilde{D}_{n, n}^{(k, m)}\right\|^{2}\right]}{E\left[\left\|D_{n, n}^{(k, m)}\right\|^{2}\right]}
$$

and it can be shown that the normalized MSE is related to the correlation coefficient by $\varepsilon=2(1-\rho)$.

In a TDD system, the CSI of the downlink can be estimated from the uplink. Some channel estimation techniques similar to those in the OFDM system (e.g. [21]-[23]) may be used. Here, we assume that the effect of Doppler spread (or channel variation) is the main source of errors. Assume the carrier frequency $f_{c}=2 \mathrm{GHz}$ and the time separation between uplink and downlink slot is $5 \mathrm{~ms}$ which is the same as the time separation in DECT [17]. By using $\rho=J_{0}\left(2 \pi f_{m} \tau\right)$ [18], where $J_{0}()$ denotes the Bessel function of the first kind, $f_{m}$ is Doppler spread and $\tau$ is the time separation between uplink and downlink transmission, the corresponding normalized MSE $\varepsilon$ when the Doppler spread, $f_{m}$, equals to 10,20 and $40 \mathrm{~Hz}$ is about $5 \%, 20 \%$ and $80 \%$, respectively. The normalized MSE is too large for $f_{m} \geq 20 \mathrm{~Hz}$ because there is more than $20 \%$ normalized MSE, so that it is necessary to increase channel estimation accuracy by some methods in order to have a good performance in our MIMOpreFDE system.

In order to obtain more accurate downlink channel estimates, the channel can be predicted by fitting a curve to the complex gain with the mean least squares (MLS) criterion [19]. By using this method, for a particular frequency, the downlink channel estimate can be given by $D_{n, n}^{(k, m)}(t)=\mathbf{t}^{T} \mathbf{a}$, where $\mathbf{t}=\left[\begin{array}{llll}1 & t & \cdots & t^{Q-1}\end{array}\right]^{T}$ is the time vector. $t$ is the time instant and $Q-1$ is the order of the polynomial fitting. The coefficient vector $\mathbf{a}=\left[\begin{array}{llll}a_{0} & a_{1} & \cdots & a_{Q-1}\end{array}\right]^{T}$ is chosen such that the square error between the uplink and downlink channel at different timing instants is minimized [20]. We refer to the fitting when $Q=2$ as First Order Prediction and to the fitting when $Q=3$ as Second Order Prediction. We demonstrate the improvement in performance of this prediction technique numerically in Section VI.

\section{B. Computational Complexity}

MIMO systems tend to have high complexity and therefore, it is useful to provide an estimate of the computational complexity of our transmit MIMO scheme. We approximate the complexity analysis by only counting the number of multiplications.

We separate the computational complexity into two parts. One is the initialization 
complexity for each data packet and the other is the processing complexity for each symbol. The initialisation complexity mainly includes computing the transmit matrix, which is approximately $M^{2} K+M K^{2}+O\left(K^{3}\right)$ multiplications for each sub-carrier, where $O\left(K^{3}\right)$ represents the number of multiplications needed to compute the matrix inverse (in the order of $K^{3}$ ). The processing complexity mainly includes computing the transmit signal by multiplying the transmitted symbols with the transmit matrix, which corresponds to a $M \times K$ matrix-vector multiplication per $K$ symbols and is equal to $M$ multiplications per symbol. In addition, extra processing complexity is added by the $K$ FFTs and $M$ IFFTs at the transmitter. Finally, note that the complexity of our proposed system is similar to that of an uncoded MIMO OFDM system with linear transmit pre-processing [21].

\section{Simulation Results}

In this section, the performance of the proposed system, the MIMO-preFDE System, is investigated by computer simulation. In the simulation, QPSK modulation is utilized and a 64-point FFT and IFFT are used for the frequency domain processing. For every 64 data symbols, 16 symbols of cyclic prefix are appended. These 80 symbols (data plus cyclic prefix) are transmitted in a time slot duration of $4 \mu \mathrm{s}$, similar to HiperLAN/II and IEEE802.11a. Unless otherwise mentioned, for simulation simplicity, a 2-ray frequency selective channel model with equal power delay profile is assumed and the time delay between the two rays is assumed to be one symbol. It is assumed that the channel is quasi-static for each data block. In the Monte-Carlo simulation, 10,000 independent data packets, each of which consists of 100 uncoded data blocks, are transmitted with independent channel. On the other hand, in the Gaussian approximation simulation, 10,000 independent channels with the conditional bit error probability given in (33) are used to obtain each BER. Throughout this section, we define the average $E_{\mathrm{b}} / N_{0}$ as the average total transmit energy per bit over the noise variance at each received antenna.

Figure 4 provides performance comparisons of the Monte-Carlo simulation and the Gaussian approximation derived in Section IV. Three configurations are shown when the number of transmit antennas is 2, 3 and 4, the number of receive antennas is 2 and the data rate is 4 bit/dimension (bit/Dim). It can be observed that the Gaussian 
approximation provides very good agreement with the Monte-Carlo simulation. Also, the results show that the performance improves when more antennas are used.

We also compare the performance of our MIMO-preFDE system with that of a MIMO OFDM system without coding. In the MIMO OFDM system, linear transmit pre-processing by channel inversion (or transmit zero forcing) [13], [21], is performed at the transmitter for each subcarrier. This transmit zero forcing is similar to the transmit zero forcing for flat fading in [12] for a single subcarrier. Notice that our MIMO-preFDE system has space-frequency transmit processing, while the MIMO OFDM system has space only transmit processing. Results are provided in Figure 5 when the number of transmit antennas ranges from 4 to 6 , the number of receive antennas is 4 , and the data rate is $8 \mathrm{bit} / \mathrm{Dim}$. We can see that our proposed MIMOpreFDE scheme outperforms the MIMO OFDM (denoted by MIMO-OFDM-preZF) significantly. Note that the performance of our MIMO-preFDE with $M=4$ is almost the same as that of the MIMO-OFDM-preZF with $M=5$. This is because our scheme not only can equalize the channel, but also can obtain multipath or frequency diversity of the channel. Note that as discussed in Section V-B, these two schemes have similar complexity.

The capability of achieving multipath or frequency diversity of the channel in the proposed MIMO-preFDE can be further demonstrated in Figure 6, where performance comparisons with three different power delay profiles: 2-ray with equal power delay profile, 7-ray with equal power delay profile, and exponential power delay profile with twelve paths and rms delay spread of two symbol duration, are provided. The time delay between the neighbour rays is assumed to be one symbol. The data rate is 4 bit/Dim. We can see that the diversity order in a 7-ray channel is larger than that in the 2-ray channel and is almost the same as that in the exponential power delay profile. Therefore, the proposed MIMO-preFDE scheme has greater advantage in severe multipath environments.

Figure 7 provides sample results for the MIMO-preFDE systems when $E_{\mathrm{b}} / N_{0}$ is equal to 10 and $15 \mathrm{~dB}$, and the data rate is $4 \mathrm{bit} / \mathrm{Dim}$ for the normalized delay spread ranging from 0 to 2 (the normalized delay spread, $d$, is defined as the root mean square (rms) delay spread over the symbol duration). The exponential power delay profile with twelve paths is assumed. As we expect, the performance improves as the 
normalized delay spread increases. In particular, for $M=3, K=2$ and $E_{\mathrm{b}} / N_{0}=10$ $\mathrm{dB}$, we can observe that the average BER when the normalized delay spread $d=2$ decreases in the order of 100 times compared to the flat fading channel, where $d=0$. Again, this is because we achieve multipath or frequency diversity of the channel. The larger the delay spread, the more the multipath diversity can be attained.

In Figure 8, the performance of the MIMO-preFDE system with a single receive antenna ( $K=1$, we refer to this as MISO-preFDE system) is compared with that of an OFDM system without coding. The number of transmit antennas ranges from 1 to 4 and data rate is 2 bit/Dim. In the OFDM system, transmit maximum ratio combining (MRC) pre-processing is used for each sub-carrier at the transmitter. This transmit MRC scheme pre-processes the data before transmission by multiplying the complex conjugate of the channel [10], [11]. Since there is only one data stream transmitted, no other multiple access technique is needed. We can observe that our proposed MISOpreFDE scheme outperforms the OFDM with MRC (denoted by MISO-OFDMpreMRC). Again, this is because of the achieved multipath or frequency diversity of the channel. Note that this MISO-preFDE system is a special case of the MIMOpreFDE system.

In Figure 9, we provide sample performance comparisons of MIMO-preFDE systems with different configurations, including the number of receive antennas $(K)$ being 2 (data rate of 4 bit/Dim) and 4 (data rate of 8 bit/Dim). Three different cases are investigated for a particular number of receive antennas including the number of transmit antennas $M=K, K+1$, and $K+2$. We can observe that for all three cases $(M=K$, $M=K+1$ and $M=K+2$ ) the configuration with $K=4$ (data rate of $8 \mathrm{bit} / \mathrm{Dim}$ ) outperforms the configuration with $K=2$ (data rate of $4 \mathrm{bit} / \mathrm{Dim}$ ). This shows an interesting behaviour that the MIMO-preFDE has greater advantage with more receive antennas when the number of transmit antennas increases accordingly. Note that the number of data streams increases as the number of receive antennas increases. This is similar to a system with multiple users, each with single receive antenna to retrieve a dedicated data sub-stream. When the number of "users" (receive antennas) and the number of transmit antennas increase simultaneously, the performance remains almost the same in flat fading channels. This demonstrates the advantage of our scheme in frequency selective channels that the data rate as well as the BER performance improve when more receive antennas as well as transmit antennas are used. 
The performance degradation for different configurations when channel estimation error occurs can be observed from Figure 10, where $E_{\mathrm{b}} / N_{0}=10 \mathrm{~dB}$. Two configurations, namely $(2,2)$ and $(3,2)$, are investigated. We can see that the average BER increases as the normalized MSE, $\varepsilon$, increases. In particular, the performance is unacceptable when the normalized MSE is larger or equal to $5 \%$ and we can conclude that the performance of the MIMO-preFDE systems is fairly sensitive to the channel estimation errors. Sample results for channel prediction (see section V-A for details) are also provided in Figure 10, where the First Order Prediction utilizes the uplink channel estimates at time instant $t \mathrm{~ms}$ and $t+10 \mathrm{~ms}$ to estimate the downlink channel at time instant $t+15 \mathrm{~ms}$, while the Second Order Prediction utilizes the uplink channel estimates at time instant $t \mathrm{~ms}, t+10 \mathrm{~ms}$, and $t+20 \mathrm{~ms}$ to estimate the downlink channel at time instant $t+25 \mathrm{~ms}$. We can see that the performance is improved. Moreover, the performance of the Second Order Prediction (dot lines) is better than that of the First Order Prediction (dash-dot lines). This is because better channel accuracy is obtained by using the prediction methods. As long as the uplink channel estimates and the estimating downlink channel are highly correlated, the channel estimation accuracy can be improved by higher order MLS prediction. Hence, channel prediction by MLS algorithm can be a potential method for solving the problem caused by Doppler spread.

\section{Conclusions}

In this paper, we have introduced a transmit MIMO scheme with frequency domain pre-equalization for a multipath or frequency selective channel, in which MIMO processing in the frequency domain is performed at the transmitter so that the receiver only requires limited processing. The optimal transmit matrix is derived by minimizing the mean square errors (MMSE). To provide a better understanding of this system, we have also derived an expression for the signal to interference-plusnoise ratio (SINR) and error probability based on the Gaussian approximation of the interference term, which has relatively accurate bit error rate estimates. Some important associated issues, such as the channel errors and the computational complexity, are investigated. It has been demonstrated by the numerical results that the performance of our proposed scheme improves when compared to other transmit 
MIMO schemes. In particular, it shows that multipath or frequency diversity of the channel is attained in our scheme.

\section{References}

[1] M. V. Clark, "Adaptive frequency-domain equalization and diversity combining for broadband wireless communications," IEEE Journal on Selected Areas in Communications, Vol. 16, No. 8, pp. 1385-1395, Oct. 1998.

[2] D. Falconer, S. L. Ariyavisitakul, A. Benyamin-Seeyar, and B. Eidson, "Frequency domain equalization for single-carrier broadband wireless systems," IEEE Communications Magazine, pp. 58-66, April 2002.

[3] IEEE 802.16ab-01/01, Air Interface for Fixed Broadband Wireless Access Systems Part A: Systems between 2 and $11 \mathrm{GHz}$, June 2001.

[4] G. J. Foschini and M. J. Gans, "On limits of wireless communications in a fading environment when using multiple antennas," Wireless Personal Commun., vol. 6, no. 3, pp. 311-335, Mar. 1998.

[5] G. D. Golden, G. J. Foschini, R. A. Valenzuela, and P. W. Wolniansky, "Detection algorithm and initial laboratory results using V-BLAST space-time communication architecture," IEE Electronics Letters, vol. 35, no. 1, pp. 14-16, Jan. 1999.

[6] Z. Xu, and R. D. Murch, "Performance analysis of maximum likelihood detection in a MIMO antenna system," IEEE Transactions on Communications, vol. 50, pp. 187-191, Feb. 2002.

[7] H. Gao, P. J. Smith, and M. V. Clark, "Theoretical reliability of MMSE linear diversity combining in Rayleigh-fading additive interference channels," IEEE Transactions on Communications, vol. 46, pp. 666-672, May 1998.

[8] H. Sampath, P. Stoica, and A. Paulraj, "Generalized linear precoder and decoder design for MIMO channels using the weighted MMSE criterion," IEEE Transactions on Communications, vol. 49, pp. 2198-2206, Dec. 2001.

[9] A. Scaglione, P. Stoica, S. Barbarossa, G. B. Giannakis, and H. Sampath, "Optimal designs for space-time linear precoder and decoders," IEEE Transactions on Signal Processing, vol. 50, pp. 1051-1064, May 2002.

[10] A. F. Naguib, A. Paulraj, and T. Kailath, "Capacity improvement with basestation antenna arrays in cellular CDMA," IEEE Transactions on Vehicular Technology, vol. 43, pp. 691-698, Aug. 1994. 
[11] R. L. Choi, K. B. Letaief, and R. D. Murch, "MISO CDMA transmission with simplified receiver for wireless communication handsets," IEEE Transactions on Communications, vol. 49, pp. 888-898, May 2001.

[12] D. Gerlach, and A. Paulraj, "Adaptive transmitting antenna arrays with feedback," IEEE Signal Processing Letters, vol. 1, pp. 150-152, Oct. 1994.

[13] P. Vandenameele, S. Thoen, M. Engels, and H. D. Man, "A combined OFDM/SDMA approach for WLAN," VTC'99, pp. 1712-1716.

[14] G. G. Raleigh, and J. M. Cioffi, "Spatio-temporal coding for wireless communication," IEEE Transactions on Communications, vol. 46, pp. 357-366, March 1998.

[15] J. Proakis, Digital Communications, New York; McGraw-Hill, fourth edition.

[16] A. Papoulis, Probability, Random Variables, and Stochastic Process, New York, McGraw-Hill Inc., 1991.

[17] O. Momtahan, and H. Hashemi, "A comparative evaluation of DECT, PACS, and PHS standards for wireless local loop applications," IEEE Personal Communications, vol. 7, pp. 35 -41, June 2000.

[18] J. D. Parsons, The Mobile Radio Propagation Channel, John Wiley \& Sons, 1992.

[19] L. L. Scharf, Statistical Signal Processing: Detection, Estimation, and Time Series Analysis, Addison Wesley, 1991.

[20] R. L. Choi, and R. D. Murch, "MIMO transmit optimization for wireless communication," IEEE International Workshop on Electronic Design, Test and Applications (DELTA 2002), Christchurch, New Zealand, pp33-37, 29-31 January 2002.

[21] P. Vandenameele, Space Division Multiple Access for Wireless Local Area Networks, Kluwer Academic Publishers, 2001.

[22] Y. G. Li, N. Seshadri, and S. Ariyavisitakul, "Channel Estimation for OFDM Systems with Transmitter Diversity in Mobile Wireless Channels," IEEE JSAC, vol. 17, pp. 461-471, March 1999.

[23] Y. G. Li, "Simplified channel estimation for OFDM systems with multiple transmit antennas," IEEE Transactions on Wireless Communications, vol. 1, pp. 67-75, Jan. 2002. 

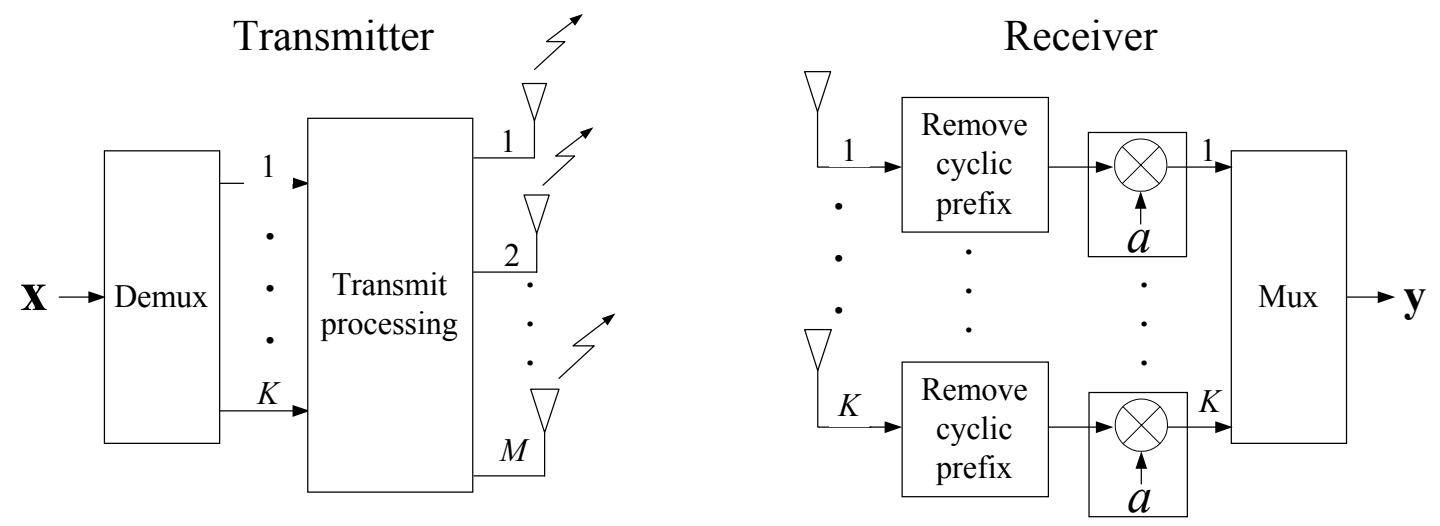

Figure 1. Configuration of the proposed MIMO-preFDE system

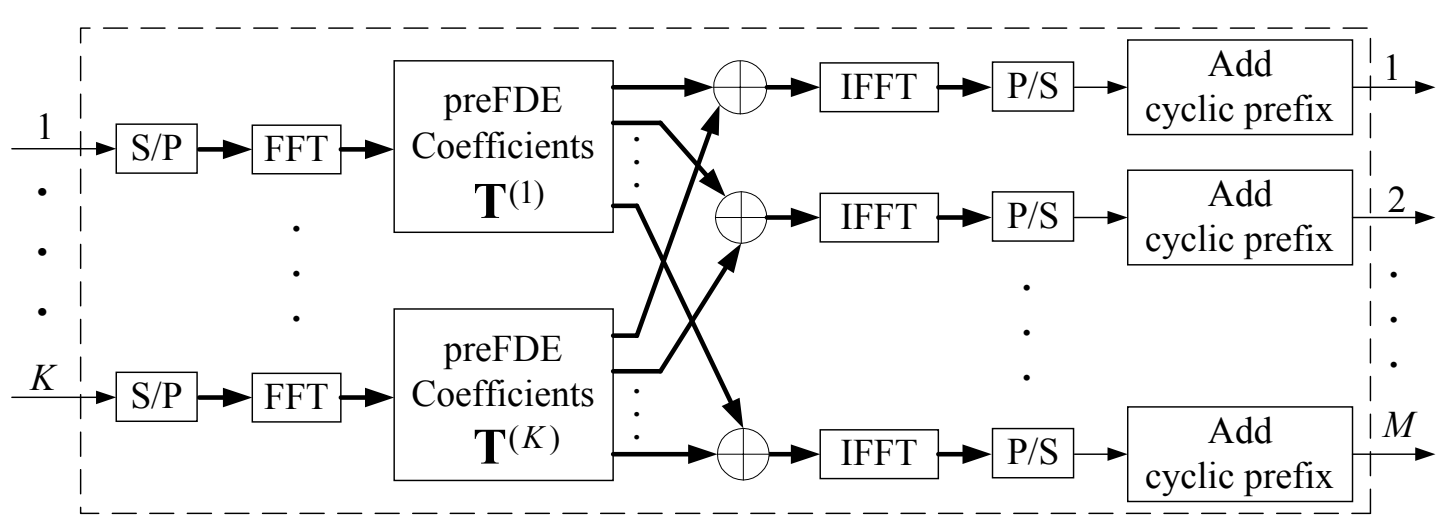

Figure 2. Block Diagram of transmit processing at the transmitter of the proposed MIMO-preFDE system 


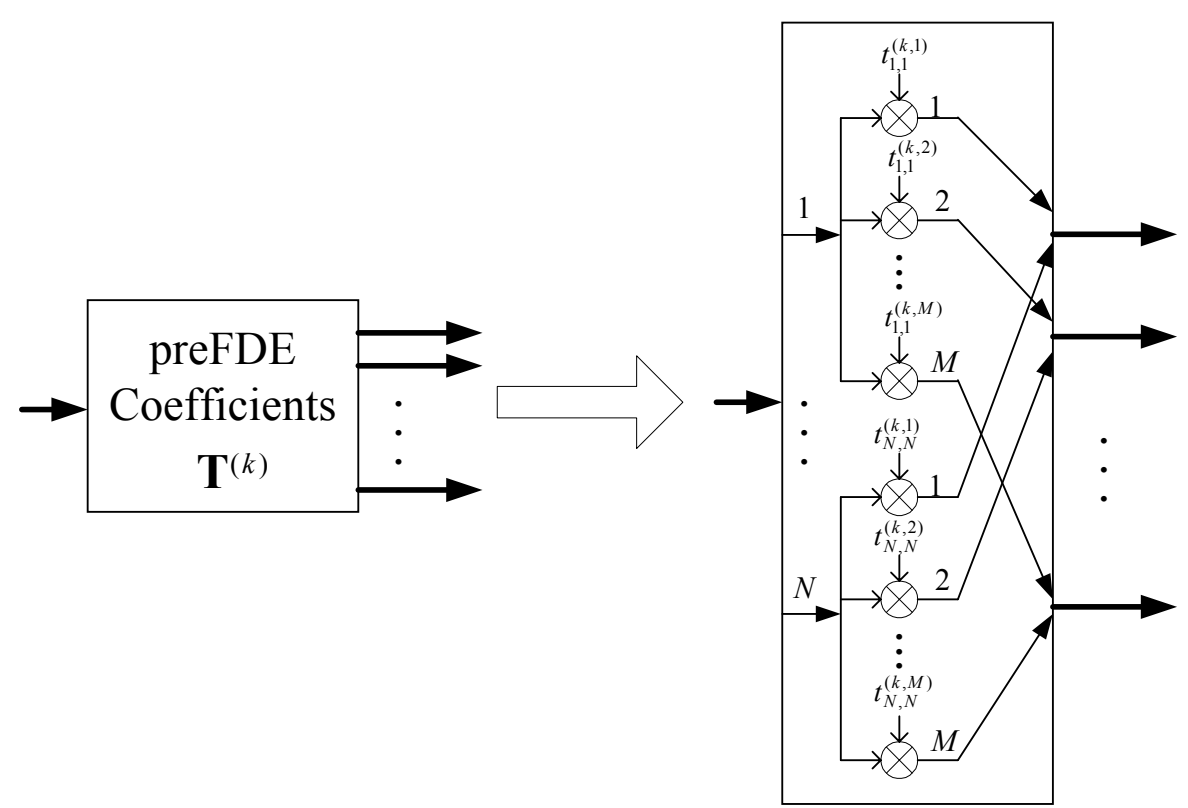

Figure 3. Transmit coefficient matrix decomposition for a particular sub-stream $(\otimes$ represents multiplication of a complex coefficient $t_{n, n}^{(k, m)}=S_{n, n}^{(k, m)} / a$ )

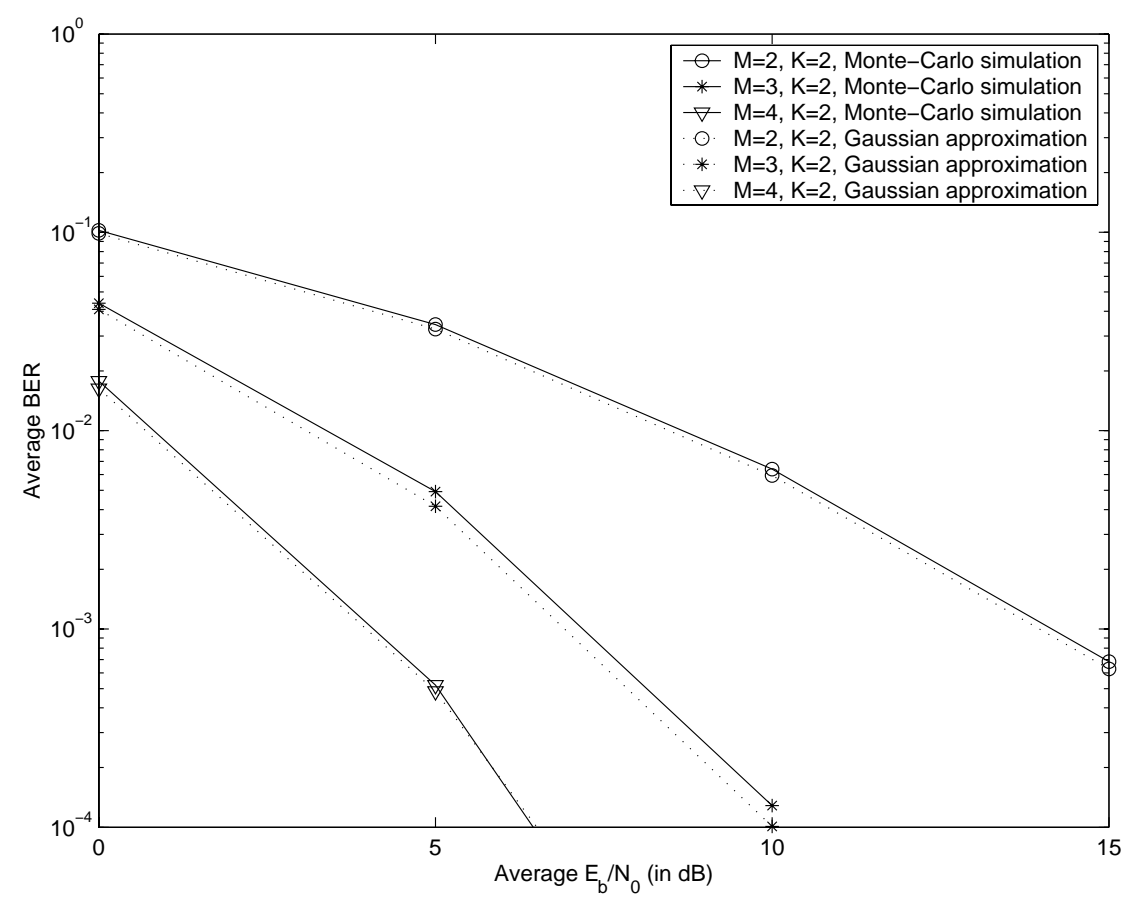

Figure 4. Performance comparisons between the Monte-Carlo simulation results and Gaussian approximation simulation results for the data rate of $4 \mathrm{bit} / \mathrm{Dim}$ 


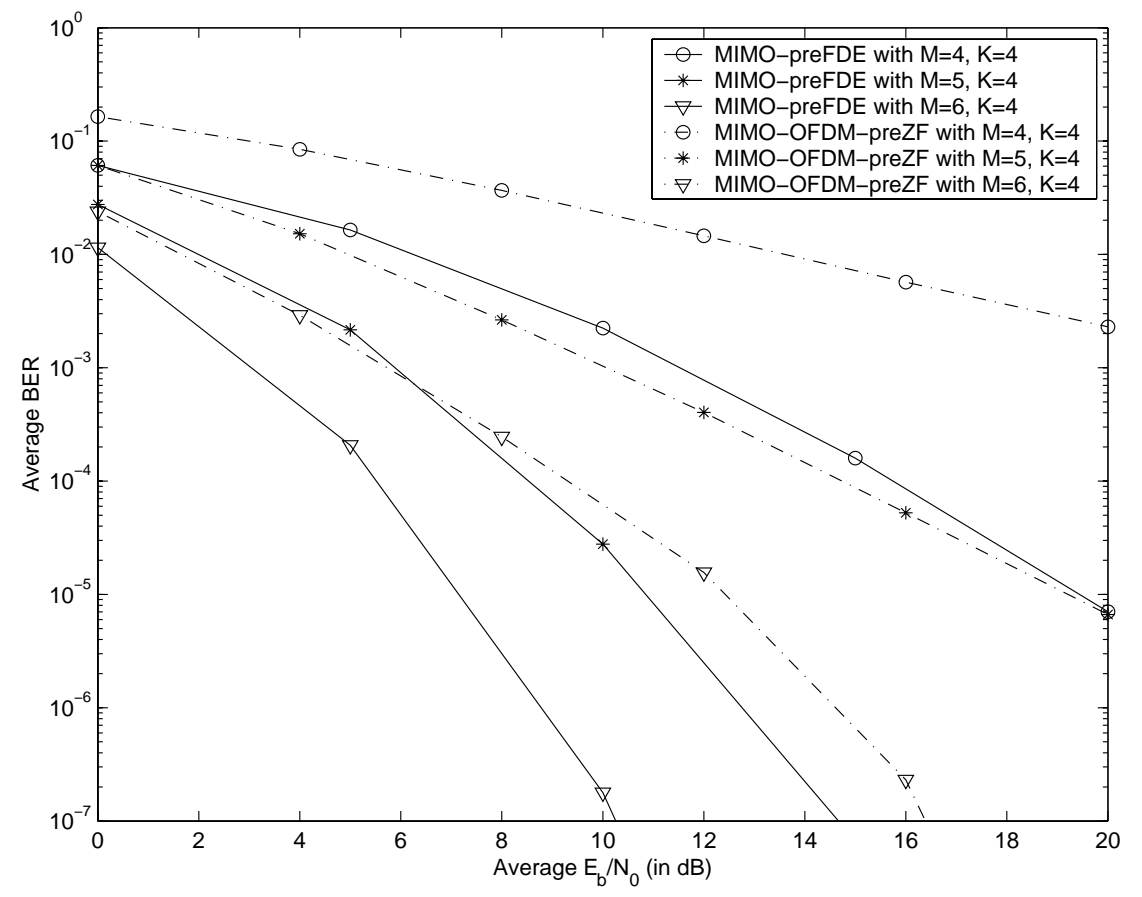

Figure 5. Performance comparisons between MIMO-preFDE systems and MIMOOFDM-preZF systems for the data rate of $8 \mathrm{bit} / \mathrm{Dim}$

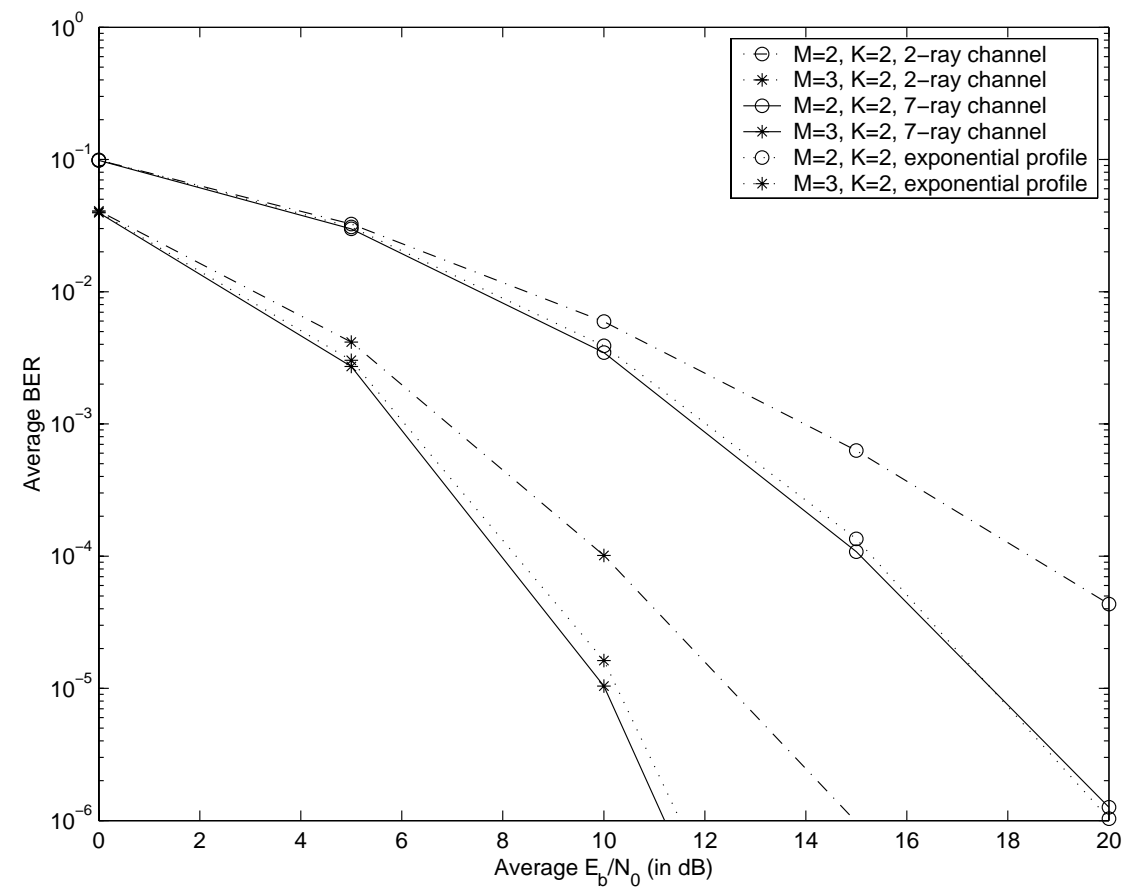

Figure 6. Performance comparisons for different power delay profiles when data rate is $4 \mathrm{bit} / \mathrm{Dim}$ 


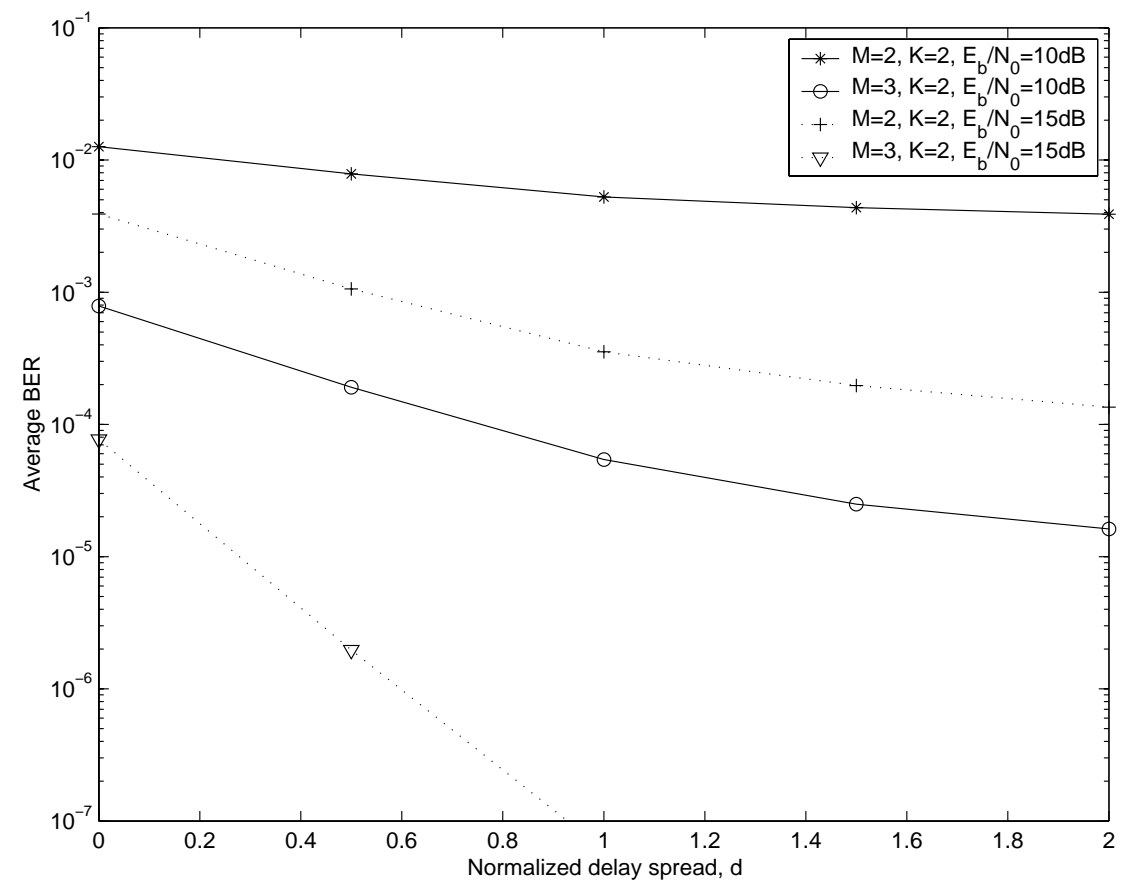

Figure 7. Performance of MIMO-preFDE systems verses normalized delay spread for $E_{b} / N_{0}$ of 10 and $15 \mathrm{~dB}$, and data rate of $4 \mathrm{bit} / \mathrm{Dim}$

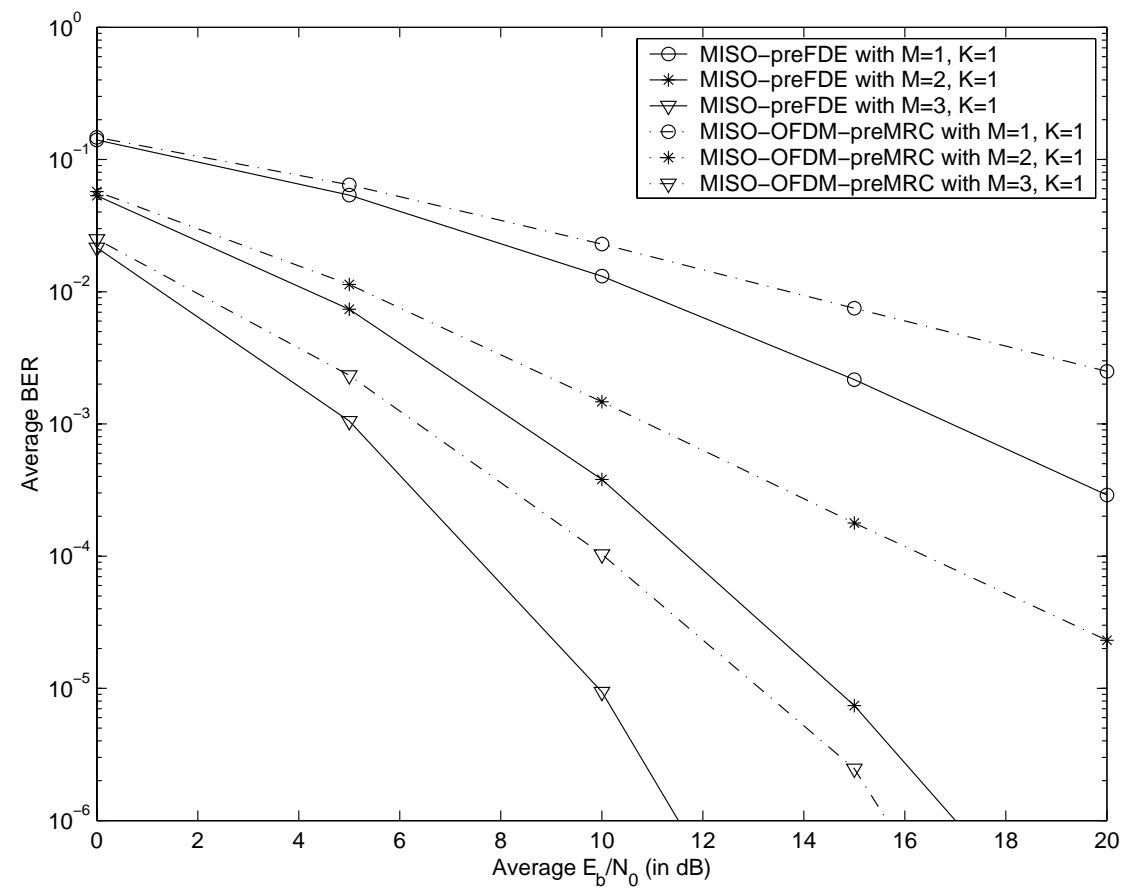

Figure 8. Performance comparisons between MISO-preFDE systems and OFDM systems with data rate of $2 \mathrm{bit} / \mathrm{Dim}$ 


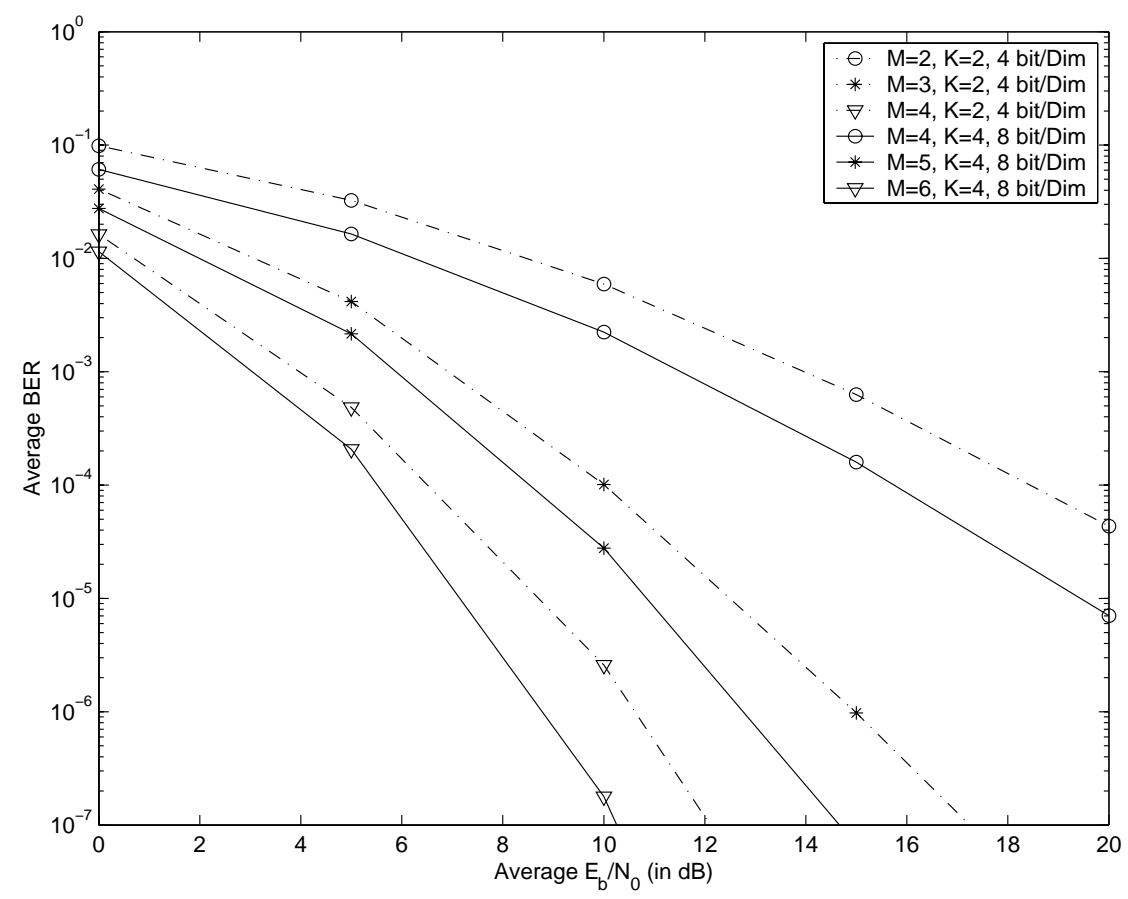

Figure 9. Performance comparisons of MIMO-preFDE systems with different configurations

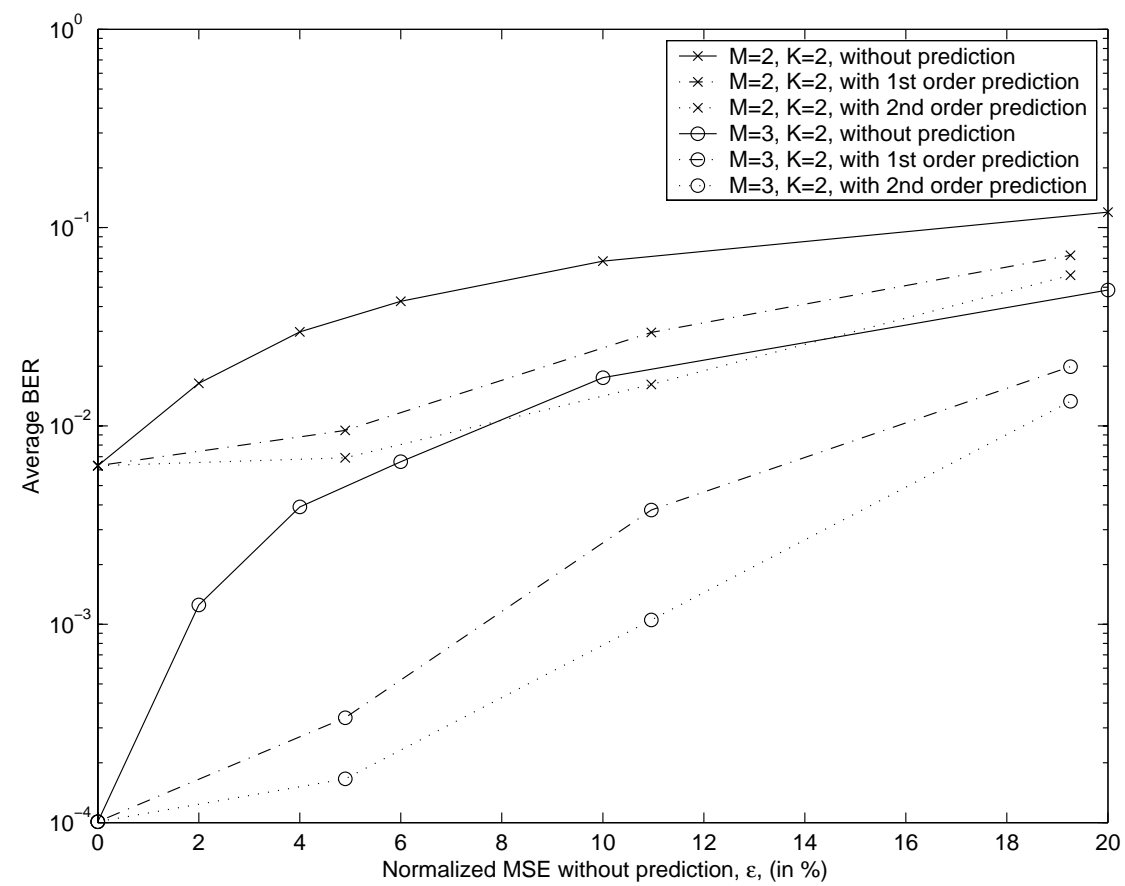

Figure 10. Performance of the MIMO-preFDE systems when channel prediction is used to estimate the downlink channel 